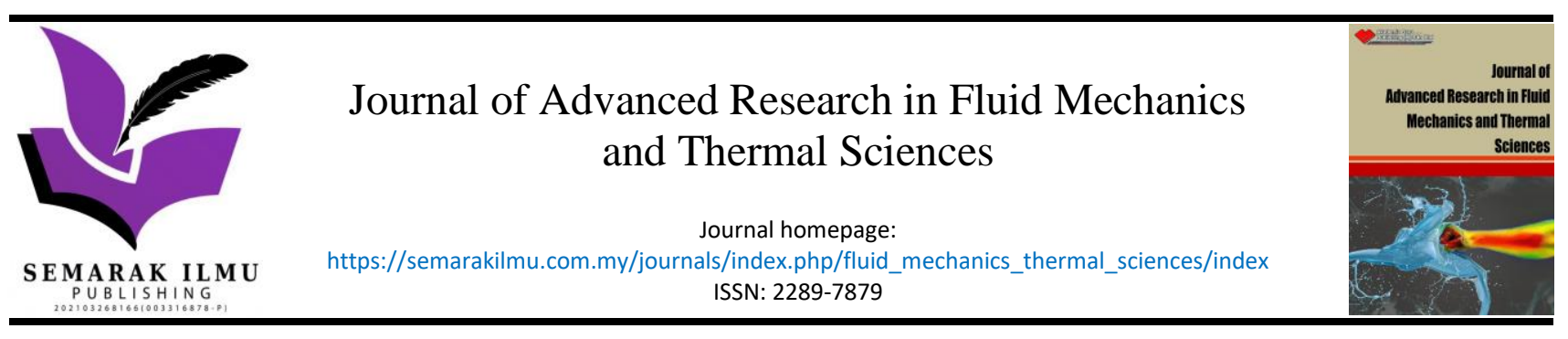

\title{
Influence of Boundary Roughness on Magnetohydrodynamic Kelvin- Helmholtz Instability in Couple-stress Fluid
}

\author{
Priya M. Gouder ${ }^{1}$, Praveen I. Chandaragi ${ }^{2,},{ }^{*}$, Krishna B. Chavaraddi $^{3}$, G. B. Marali ${ }^{2}$ \\ Department of Mathematics, KLE'S Dr. M. S. Sheshgiri College of Engineering and Technology, Belagavi-590008, Karnataka, India \\ Department of Mathematics, KLE Technological University, Hubli-580031, Karnataka, India \\ Department of Mathematics, S.S. Government First Grade College and P.G. Studies Center, Nargund-582207, Karnataka, India
}

ARTICLE INFO

\section{Article history:}

Received 6 August 2021

Received in revised form 23 September 2021

Accepted 25 September 2021

Available online 29 October 2021

\section{Keywords:}

Kelvin-Helmholtz instability; boundary roughness; porous layer; Couple-stress fluids; magnetic field

\section{ABSTRACT}

\begin{abstract}
The Kelvin-Helmholtz instability (KHI) occurs at the interface amongst two fluids, which are in relative motion with a common boundary. The growth rate of waves occurs whenever the relative velocity is greater as compared with the critical relative velocity. In the present paper, the influence of boundary roughness on $\mathrm{KHI}$ under the impact magnetic field in a couple-stress fluid layer bounded by a rigid surface at the lower side and upper side by a fluid saturated porous layer. Using suitable surface and boundary conditions, we have derived the dispersion relation and results are depicted graphically. As observed in presence of sharp interface, magnetic field exhibits stabilizing effect however, destabilizing effect is shown by the buoyancy force on $\mathrm{KHI}$. Also, noted that the growth rate of interface reduces, as there is a rise in roughness parameter value.
\end{abstract}

\section{Introduction}

The basic instabilities affecting an interface between two-fluid systems, is KHI. One of the ideal situations is where one layer of heavier fluid underlying another of lighter fluid when both are flowing horizontally with different velocities in same direction. Helmholtz [1] and Thomson [2] developed this theory, which has developed as a standard foundation for fluid mechanics and basic theory of instabilities over the composite fluid layers as established in numerous studies. Lamb [3], Turner [5], Kundu [8] and Scorer [11] are few books to cite. Comparing to any other type of fluid instability, the investigations carried out into this instability is perhaps with far more depth.

A huge literature is generated, due to the significance of $\mathrm{KHI}$ in parallel flows occurring in laboratory, astrophysical or geophysical systems that were known many years back. In hydrodynamics, the study of $\mathrm{KHI}$ has a vast history especially with analysis of basic linear stability related to magneto-hydrodynamic (MHD) KHI was considered from earlier [4]. Presently, there is a developing literature for nonlinear evolution magentohydrodynamic KHI starting from various possible initial configurations, in two dimensions mostly in the earlier evolution stages. It is seen

\footnotetext{
* Corresponding author.

E-mail address: praveen.chandargi@kletech.ac.in
} 
that strong magnetic fields due to their tension stabilize the KHI. Recently, it is emphasized that the significant potential for considerable weaker fields to transform the nonlinear unsteadiness i.e., to alter the consequent flow.

Malik and Singh [7] at the interface of two superposed Ferro fluids, which were moving parallel to the common interface with uniform speeds under a tangential magnetic field, carried out the investigation of nonlinear $\mathrm{KH}$ properties of $(2+1)$ dimensional wave propagating packets. Derived nonlinear equation manages the growth of the interface amplitude. $\mathrm{KHI}$ is very commonly observed among environmental fluids, which are simultaneously subjected to the stabilization because of density stratification and destabilization due to velocity shear [9]. Under the influence of a tangential magnetic field on nonlinear KH instability, El-Dib [10] has analyzed the consequences of a time-dependent acceleration. By keeping the main objective as; the evolution of $\mathrm{KHI}$ can be reduced by using porous layer, a simple theory is established based on Stokes and lubrication approximations is applied, which follows Babchin et al., [6] and Rudraiah et al., [12]. Bhatia and Sharma [13] examined the permeability effects due to porous medium and surface tension on KHI on a two-fluid system which are superposed and viscous under uniform vertical magnetic field. The fluid considered in the above studies is Newtonian fluid.

Electrorheological $\mathrm{KHI}$ of a fluid sheet by considering the gravitational steadiness of an electrified Maxwellian fluid is deliberated by El-Dib and Matoog [14]. On the interfaces of the fluid sheet, surface charges were produced by the field. In this study, the weak significances of viscoelastic fluids are considered for mathematical simplification due to the complexity of the problem.

In recent years, significant effort is put to understand the occurrence of couple-stress effects with fluid saturated porous media in non-Newtonian fluid flow. This field is of special interest as there are many applications in various fields. Such as, to improve oil recovery competency from water flooding projects in oil reservoir with displacing non-Newtonian fluids by mobility control. Understanding the effect of couple-stress in case of displaced and displacing non-Newtonian fluids in an oil displacement mechanism has become essential. The parallel flow moving through porous media for the fluids having different viscosity, density and elasticity are involved in most of the technological processes. These flows are observed in chemical industry particularly in packed bed reactors, petroleum engineering, boiling in porous media etc. The interface may become unstable due to a significant rise in the resistance on the flow, resulting into dry out in boiling porous media due to flooding in counter current packed chemical reactors. Similar to this, in petroleum production these discontinuities yield to emulsion formation. Therefore, in above processes to analyze the limiting operation situations we should have the knowledge of onset of instability conditions.

In the two-dimensional system immersed in presence of uniform horizontal magnetic field Khan et al., [15] discuss KHI in two superposed highly viscous conducting liquids of uniform densities by taking into deliberation of surface tension effects. The porosity, viscosity and surface tension effects are found to show stabilizing impact on the evolution of unstable mode, whereas a destabilization on the system by streaming velocity is exhibited.

On the basis of two simple models related to exchange of energy occurring amongst the superposed fluids, Joshi et al., [16] investigated the root cause of KHI. In determination of minimal relative speed causing the instability is due to surface tension and density of fluids. In case of dielectric and ferro-fluids, the volume force employed by magnetic and electric field gradients is also discussed. Chandrashekara and Rudraiah [17] concerned with the study of the Electrorheological Kelvin-Helmholtz Instability (EKHI) at the interface between a poorly conducting couple stress fluid saturated porous layer which is in relative motion with a poorly conducting 
couple stress fluid in a thin shell in the presence of a transverse electric field and laser radiation. Chavaraddi et al., [18] deliberated the impact of boundary roughness on $\mathrm{KHI}$ in couple-stress fluid layer. Effects due to surface roughness in the existence of magnetic field on $\mathrm{KHI}$ are investigated [19].

Discontinuity of a two fluid system which are immiscible, non-viscous, superposed, counter streaming, electrically conducting with lower fluid layer heavier than the upper fluid layer in presence of magnetic field is numerically considered by Sharma and Gupta [20]. Using linear theory by normal mode analysis, accurate solutions for stress free bounding surfaces related to eigenvalue problem were obtained. On the evolution of the unsteady perturbation of the physical system, the significance of velocity and magnetic field of streaming fluids is examined. Magnetic field has found to have insignificant stabilizing effect whereas a great extent of stabilization is due to velocity of the counter-streaming fluids. The magnetic field effects on $\mathrm{KHI}$ in a couple-stress fluid layer bounded below by a rigid surface and above by a porous layer is analyzed by Chavaraddi et al., [21].

The stability of pressure-driven parallel shear flow in a horizontal layer of couple stress fluid saturated porous medium is investigated using a classical linear stability theory by Shankar et al., [22]. A modified Orr-Sommerfeld equation is derived and solved numerically using the Chebyshev collocation method. Shankar et al., [23] have conducted to analyze the influence of a uniform horizontal magnetic field on the stability of buoyancy driven parallel shear flow in a differentially heated vertical layer of an electrically conducting couple stress fluid; a type of non-Newtonian fluid. Within the framework of linear stability theory, the resulting complex generalized eigenvalue problem is solved numerically using the Chebyshev collocation method with QZ algorithm.

$\mathrm{KHI}$ of a parallel shear flow system consisting of hyperbolic tangent velocity profile at high Reynolds number is simulated Liu et al., [24]. In the study, fluid with low viscosity, which is perfectly conducting is considered. There is a variation in strength of applied magnetic field from weak to strong. On the shear flow with short wave perturbations, author observed that magnetic field has a stabilizing influence, due to two parts: splitting impact arising from transverse magnetic pressure and impact due to anti-bonding magnetic tension. Shankar et al., [25] discussed the effect of a uniform vertical magnetic field on the stability of pressure-driven flow of an electrically conducting non-Newtonian fluid in an isothermal channel is numerically investigated using the Chebyshev collocation method. It is found that increasing $M$ has a stabilizing effect on the system while an increase in the couple stress parameter shows two-fold deeds. Recently, in the work of Naveen Kumar et al., [26], the effect of Coriolis force is explored on convective instability of a doubly diffusive incompressible couple stress fluid layer with gravity acting downward. The implication of couple stresses on each of the aforementioned anomalies is clearly brought out. Very recently Farahani et al., [27] investigated the effect of magnetic field on temperature field and nanofluid using finite volume method. Also, the impact of streamwise steps and cavities geometry on supersonic turbulent boundary layer is presented by Ch'ng et al., [28]. This study involved a parametric numerical study carried out using computational fluid dynamics(CFD) software package ANSYS FLUENT 19.2 for providing a qualitative insight into the TBL and flow field distortions caused by periodic surface roughness elements in the form of steps and cavities under Mach 3.0 flow conditions.

In the present paper, the influence of boundary roughness on $\mathrm{KHI}$ in existence of magnetic field in couple-stress is studied. This paper is planned as: In Section 2, basic equations along with Maxwell's equations is established. In Section 3, equations are non-dimensionalized by following Stokes and lubrication approximations. By applying suitable surface and boundary conditions the dispersion relation is obtained in Section 4 . In addition, the cut off wave number, maximum wave 
number as well as corresponding maximum growth rate are found. As observed in graphical representations, some important interpretations are discussed in last section.

\section{Mathematical Formulation}

The physical arrangement of the problem is represented in Figure 1. A thin target shell is considered in the form of a thin film with $h$ as unperturbed thickness (Region-1) occupied with an incompressible, electrically poorly conducting, viscous, less dense fluid with density $\rho_{f}$ bounded by a rigid surface at lower side $y=0$. An incompressible poorly conducting viscous more dense fluid bounded above the light fluid with density $\rho_{p}$ saturating a dense porous layer with large extent in comparison with $h$ the shell thickness. The $x$-co-ordinate and $y$-co-ordinate spans respectively the vertical and horizontal directions. The interface existing at $y=h$ is symbolized by $\eta(x, t)$. Obviously, when the boundary between the fluids is flat then $\eta=0$ at $y=h$. The fluid is supposed to be nonNewtonian, electrically conducting, viscous as well as incompressible with velocity vector, $\overrightarrow{q_{1}}=(u, v) . \alpha_{p}$ is slip parameter at the interface and $\mu_{f}\left(\mu_{p}\right)$ represents fluid viscosity (porous medium). The stress-gradient is $\delta=g\left(\rho_{p}-\rho_{f}\right)$ which is associated with acceleration due to gravity. The disturbed boundary $\eta(x, t)$ is in the $y$-direction.

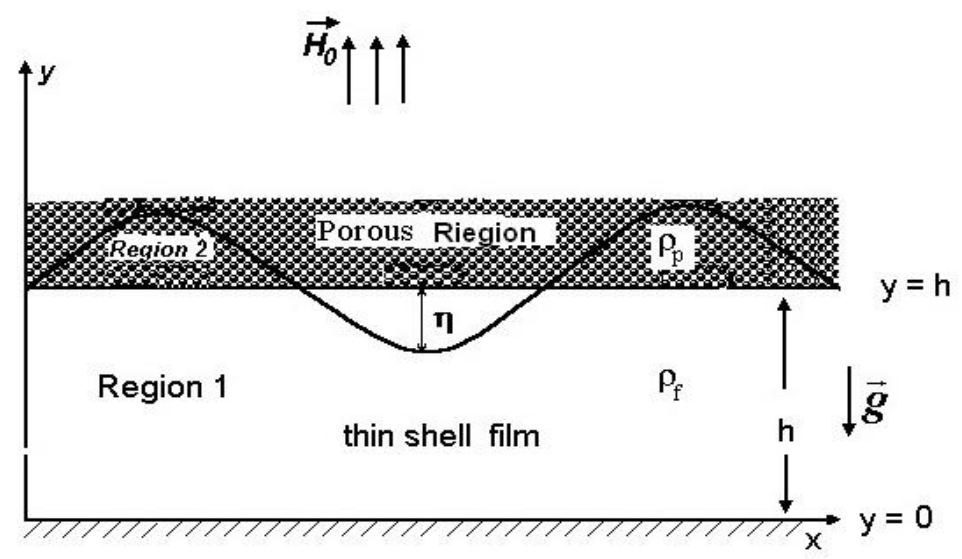

Fig. 1. Physical Configuration

The basic equations associated with clear fluid layer (Region-1) and which are related with porous layer (Region-2) given as follows:

Region-1:

$\nabla \cdot \vec{q}_{1}=0$

$\rho\left(\frac{\partial \vec{q}_{1}}{\partial t}+\left(\vec{q}_{1} \cdot \nabla\right) \vec{q}_{1}\right)=-\nabla p_{1}+\mu_{f} \nabla^{2} \vec{q}_{1}-\lambda_{f} \nabla^{4} \vec{q}_{1}+\mu_{0}\left(\vec{J}_{1} \times \vec{B}_{1}\right)$

Maxwell's Equations: 


$$
\nabla \cdot \vec{E}_{1}=0, \nabla \cdot \vec{H}_{1}=0, \nabla \times \vec{E}_{1}=-\frac{\partial \vec{B}_{1}}{\partial t}, \nabla \times \vec{H}_{1}=\vec{J}_{1}+\frac{\partial \vec{D}_{1}}{\partial t}
$$

Auxiliary equations:

$$
\vec{D}_{1}=\varepsilon_{0} \vec{E}_{1}, \vec{B}_{1}=\mu_{0} \vec{H}_{1}, \vec{J}_{1} \times \vec{B}_{1}=\sigma\left[\vec{E}_{1}+\vec{q}_{1} \times \vec{B}_{1}\right] \times \vec{B}_{1}
$$

Region-2:

$$
\overrightarrow{Q_{1}}=-\frac{k}{\mu_{f}} \frac{\partial p_{1}}{\partial x}
$$

To simplify basic equations Stokes and lubrication assumptions and electro hydrodynamic approximations are considered as follows [13]:

(i) For the liquid considered, the electrical conductivity $\sigma$ is insignificantly small, i.e., $\sigma<<1$.

(ii) The film thickness $h$ is much lesser in comparison with thickness $H$ of the denser liquid lying on the film.

i.e., $\quad h<<H$.

(iii) The surface elevation $\eta$ is supposed to be small as compared with the film thickness $h$. i.e., $\eta<<h$.

(iv) The Strauhal number $S$ in Eq. (2), is insignificantly small.

$$
\text { i.e., } S=\frac{L}{T U}<<1
$$

The above equations are non-dimensionalized using the following,

$$
u^{*}=\frac{u}{\delta h^{2} / \mu_{f}}, v^{*}=\frac{v}{\delta h^{2} / \mu_{f}}, p_{1}^{*}=\frac{p_{1}}{\delta h}, Q_{1}^{*}=\frac{Q_{1}}{\delta h^{2} / \mu_{f}}, t^{*}=\frac{t}{\delta h / \mu_{f}}, x^{*}=\frac{x}{h}, y^{*}=\frac{y}{h}
$$

By using the assumptions and approximations as specified above, also by assuming that more dense fluid in the porous layer is practically static caused by creeping flow approximation and using Eq. (6) in the Eq. (1) and Eq. (2), we get,

Region 1:

$0=\frac{\partial u}{\partial x}+\frac{\partial v}{\partial y}$

$0=-\frac{\partial p_{1}}{\partial x}+\frac{\partial^{2} u}{\partial y^{2}}-M_{0}^{2} \frac{\partial^{4} u}{\partial y^{4}}-M^{2} u$ 
$0=-\frac{\partial p_{1}}{\partial y}$

Region 2:

$Q_{1}=-\frac{1}{\sigma_{p}^{2}} \frac{\partial p_{1}}{\partial x}$

\section{Dispersion Relation}

Firstly, the velocity distribution is obtained from Eq. (8) to find the dispersion relation, by applying the boundary and surface conditions given as follows:

(i) Roughness condition:

$$
-\beta \frac{\partial u}{\partial y}=u \text { at } y=0
$$

(ii) Beaver-Joseph slip condition:

$$
\frac{\partial u}{\partial y}=-\sigma_{p} \alpha_{p}\left(u_{B}-Q_{1}\right) \text { at } y=1 \quad \text { (also } y=1, u=u_{B} \text { ) }
$$

(iii) Kinematic condition:

$$
v=\frac{\partial \eta}{\partial t} \text { at } y=1
$$

(iv) Dynamic condition:

$$
p_{1}=-\eta-\frac{1}{B_{1}} \frac{\partial^{2} \eta}{\partial x^{2}} \text { at } y=1 \text {. }
$$

The solution of Eq. (8) applying the surface and boundary conditions as given above is

$$
\begin{aligned}
& \begin{array}{l}
u=\left[C_{1} \operatorname{Cosh}\left(\alpha_{1} y\right)+C_{2} \operatorname{Sinh}\left(\alpha_{1} y\right)+C_{3} \operatorname{Cosh}\left(\alpha_{2} y\right)+C_{4} \operatorname{Sinh}\left(\alpha_{2} y\right)-\frac{P}{M^{2}}\right] \\
\text { where } P=\frac{\partial p_{1}}{\partial x}, \\
\alpha_{1}^{2}=\frac{1+\sqrt{1-4 M^{2} M_{0}^{2}}}{2 M_{0}^{2}}, \alpha_{2}^{2}=\frac{1-\sqrt{1-4 M^{2} M_{0}^{2}}}{2 M_{0}^{2}}, \\
a_{1}=\alpha_{1} \operatorname{Sinh}\left(\alpha_{1}\right)+\alpha_{p} \sigma_{p} \operatorname{Cosh}\left(\alpha_{1}\right), \quad a_{2}=\alpha_{1} \operatorname{Cosh}\left(\alpha_{1}\right)+\alpha_{p} \sigma_{p} \operatorname{Sinh}\left(\alpha_{1}\right), \\
a_{3}=\alpha_{2} \operatorname{Sinh}\left(\alpha_{2}\right)+\alpha_{p} \sigma_{p} \operatorname{Cosh}\left(\alpha_{2}\right), \quad a_{4}=\alpha_{2} \operatorname{Cosh}\left(\alpha_{2}\right)+\alpha_{p} \sigma_{p} \operatorname{Sinh}\left(\alpha_{2}\right),
\end{array}
\end{aligned}
$$




$$
\begin{aligned}
& a_{5}=\alpha_{1}^{2} \operatorname{Cosh}\left(\alpha_{1}\right), a_{6}=\alpha_{1}^{2} \operatorname{Sinh}\left(\alpha_{1}\right), a_{7}=\alpha_{2}^{2} \operatorname{Cosh}\left(\alpha_{2}\right), a_{8}=\alpha_{2}^{2} \operatorname{Sinh}\left(\alpha_{2}\right), \\
& b_{1}=\frac{1}{M^{2}}, b_{2}=\frac{1}{M^{2}}-\frac{\alpha_{p}}{\sigma_{p}}, \\
& C_{1}=\frac{P \alpha_{2}{ }^{2}\left[b_{1}\left(a_{2} a_{8}-a_{4} a_{6}\right)-b_{2} \beta\left(\alpha_{1} a_{8}-\alpha_{2} a_{6}\right)\right]}{\beta\left[\alpha_{1}^{3}\left(a_{3} a_{8}-a_{4} a_{7}\right)+\alpha_{1}{ }^{2} \alpha_{2}\left(a_{2} a_{7}-a_{3} a_{6}\right)+\alpha_{1} \alpha_{2}{ }^{2}\left(a_{4} a_{5}-a_{1} a_{8}\right)+\alpha_{2}^{3}\left(a_{1} a_{6}-a_{2} a_{5}\right)\right]+\left(\alpha_{1}{ }^{2}-\alpha_{2}{ }^{2}\right)\left(a_{4} a_{6}-a_{2} a_{8}\right)}, \\
& C_{2}=\frac{P\left\{b_{1}\left[\alpha_{1}^{2}\left(a_{3} a_{8}-a_{4} a_{7}\right)-\alpha_{2}{ }^{2}\left(a_{1} a_{8}-a_{4} a_{5}\right)\right]+b_{2}\left[\beta\left(\alpha_{1}{ }^{2} \alpha_{2} a_{5}-\alpha_{2}{ }^{3} a_{5}\right)-\left(\alpha_{1}^{2}-\alpha_{1}^{2}\right) a_{8}\right]\right\}}{\beta\left[\alpha_{1}^{3}\left(a_{3} a_{8}-a_{4} a_{7}\right)+\alpha_{1}^{2} \alpha_{2}\left(a_{2} a_{7}-a_{3} a_{6}\right)+\alpha_{1} \alpha_{2}^{2}\left(a_{4} a_{5}-a_{1} a_{8}\right)+\alpha_{2}{ }^{3}\left(a_{1} a_{6}-a_{2} a_{5}\right)\right]+\left(\alpha_{1}^{2}-\alpha_{2}^{2}\right)\left(a_{4} a_{6}-a_{2} a_{8}\right)}, \\
& C_{3}=\frac{-P \alpha_{1}^{2}\left[b_{1}\left(a_{2} a_{8}-a_{4} a_{6}\right)-b_{2} \beta\left(\alpha_{1} a_{8}-\alpha_{2} a_{6}\right)\right]}{\beta\left[\alpha_{1}^{3}\left(a_{3} a_{8}-a_{4} a_{7}\right)+\alpha_{1}^{2} \alpha_{2}\left(a_{2} a_{7}-a_{3} a_{6}\right)+\alpha_{1} \alpha_{2}^{2}\left(a_{4} a_{5}-a_{1} a_{8}\right)+\alpha_{2}{ }^{3}\left(a_{1} a_{6}-a_{2} a_{5}\right)\right]+\left(\alpha_{1}{ }^{2}-\alpha_{2}{ }^{2}\right)\left(a_{4} a_{6}-a_{2} a_{8}\right)}, \\
& C_{4}=\frac{P\left\{b_{1}\left[\alpha_{1}^{2}\left(a_{2} a_{7}-a_{4} a_{6}\right)+\alpha_{2}{ }^{2}\left(a_{1} a_{6}-a_{2} a_{5}\right)\right]-b_{2}\left[\beta\left(\alpha_{1}{ }^{3} a_{7}-\alpha_{1} \alpha_{2}{ }^{2} a_{5}\right)-\left(\alpha_{1}^{2}-\alpha_{1}^{2}\right) a_{6}\right]\right\}}{\beta\left[\alpha_{1}{ }^{3}\left(a_{3} a_{8}-a_{4} a_{7}\right)+\alpha_{1}{ }^{2} \alpha_{2}\left(a_{2} a_{7}-a_{3} a_{6}\right)+\alpha_{1} \alpha_{2}{ }^{2}\left(a_{4} a_{5}-a_{1} a_{8}\right)+\alpha_{2}{ }^{3}\left(a_{1} a_{6}-a_{2} a_{5}\right)\right]+\left(\alpha_{1}{ }^{2}-\alpha_{2}{ }^{2}\right)\left(a_{4} a_{6}-a_{2} a_{8}\right)} .
\end{aligned}
$$

By integrating Eq. (7) from $y=0$ to $y=1$ and using Eq. (15), we obtain

$$
v(1)=\left[\frac{\partial^{2} \eta}{\partial x^{2}}+\frac{1}{B_{1}} \frac{\partial^{4} \eta}{\partial x^{4}}\right] \Delta_{1}
$$

where $\Delta_{1}=\frac{C_{1}}{\alpha_{1}} \operatorname{Sinh}\left(\alpha_{1}\right)+\frac{C_{2}}{\alpha_{1}}\left[\operatorname{Cosh}\left(\alpha_{1}\right)-1\right]+\frac{C_{3}}{\alpha_{2}} \operatorname{Sinh}\left(\alpha_{2}\right)+\frac{C_{4}}{\alpha_{2}}\left[\operatorname{Cosh}\left(\alpha_{2}\right)-1\right]-\frac{1}{M^{2}}$.

Using Eq. (16) and Eq. (14), then Eq. (13), reduces to

$$
\frac{\partial \eta}{\partial t}=\left[\frac{\partial^{2} \eta}{\partial x^{2}}+\frac{1}{B_{1}} \frac{\partial^{4} \eta}{\partial x^{4}}\right] \Delta_{1} .
$$

Let us consider the solution of Eq. (17) given by Eq. (18) in the subsequent form to examine $n$ i.e., growth rate of the interface with periodic perturbation.

$$
\eta=\eta(y) e^{\{i \ell x+n t\}}
$$

here $\eta(y)$ is perturbation amplitude of the interface and $\ell$ is the wave number.

Let us substitute Eq. (18) in Eq. (17), the dispersion relation is obtained in the form 
$n=\ell^{2}\left(1-\frac{\ell^{2}}{B_{1}}\right) \Delta$

where $\Delta=-\Delta_{1}$

Also, Eq. (19) is represented as

$n=n_{b}-\ell \beta v_{a}$

where $n_{b}=\frac{\ell^{2}}{3}\left[1-\frac{\ell^{2}}{B_{1}}\right], \beta=\Delta \ell\left[1-\frac{\ell^{2}}{B_{1}}\right], v_{a}=\left(\frac{1-3 \Delta}{3 \Delta}\right)\left(1-\frac{\ell^{2}}{B_{1}}\right)$.

By setting $n=0$ in Eq. (19), $\ell_{c t}$ the cut-off wavenumber is obtained as

$\ell_{c t}=\sqrt{B_{1}}$

since $\ell$ and $\Delta$ are different from zero.

From Eq. (19), by setting $\frac{\partial n}{\partial \ell}=0$ the value of $\ell_{m}$ i.e., maximum wavenumber is established as

$\ell_{m}=\sqrt{\frac{B_{1}}{2}}=\frac{\ell_{c t}}{\sqrt{2}}$

The value of $n_{m}$ i.e., corresponding maximum growth rate is expressed as

$n_{m}=\frac{B_{1}}{4} \Delta$

In similar way by using $\ell_{m}=\sqrt{\frac{B_{1}}{2}}$, we get

$n_{b m}=\frac{B_{1}}{12}$

Therefore, maximum growth rate is given by

$$
G_{m}=\frac{n_{m}}{n_{b m}}=3 \Delta
$$


The evolution rate represented by Eq. (19) is calculated by taking different values of the parameter. The results are depicted through graphs in Figure 2 to Figure 6.

\section{Results and Discussion}

In this present work, the influence of boundary roughness on $\mathrm{KHI}$ in presence of magnetic field, which is occurring in couple-stress fluid layers bounded below by a rigid boundary and above by a porous layer is analyzed. Computations to investigate growth rate at the interface were executed for various fluid properties corresponding to Hartmann number $M$, roughness parameter $\beta$, couple-stress parameter $M_{0}$, porous parameter, $\sigma_{p}$ and Bond number, $\boldsymbol{B}_{1}$ at different wavenumbers. Graphs were plotted for non-dimensionalized evolution rate $n$ of the perturbation corresponding to the values of $\ell$ the non-dimensionalized wavenumber, only for certain cases. In agreement with the dispersion relation, all perturbed values raise exponentially in case of linear stage given by Eq. (19). The boundary between the layers at this stage attains a sinusoidal shape with small amplitude.

Based on the problem related to two-layer channel flow, the role of magnetic field is studied and it is demonstrated that either stabilization or destabilization can be obtained. For a rise in the values of Hartmann number $M$ it represents growth rates in conditions for which magnetic field sows' stabilization effect corresponding to a wide range of wavenumbers as depicted in Figure 2, where $\alpha_{p}=0.1, \sigma_{p}=4, B_{1}=0.02, \beta=3.3 \times 10^{-3}, M_{0}=0.3$. Notice that as the values of $M$ are increased from 5 to 50 there is a decrease in maximum growth rate.

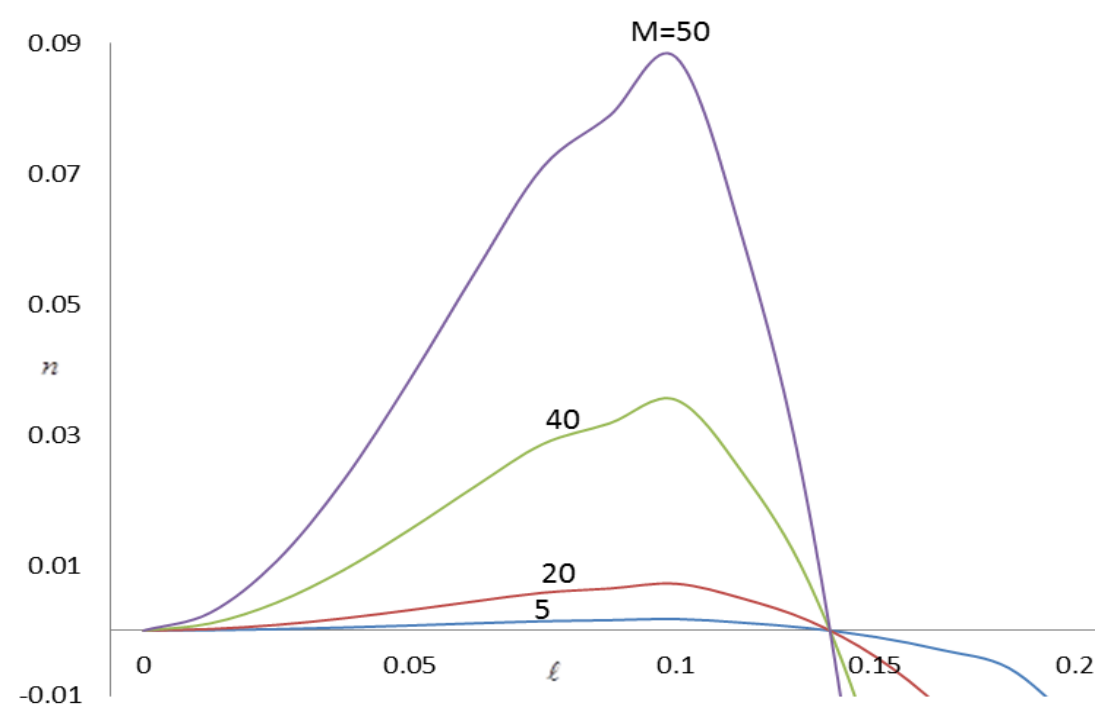

Fig. 2. Variations of Hartman Number

Growth rate versus the wavenumber is depicted in Figure 3 with fixed the value $\alpha_{p}=0.1, M=5, \sigma_{p}=4, B_{1}=0.02, \beta=3.3 \times 10^{-3}$ for different values of $M_{0}$. As there is a rise in couple-stress parameter value results into decrease in maximum growth rate this is due to the action of the body couples related to the system. 


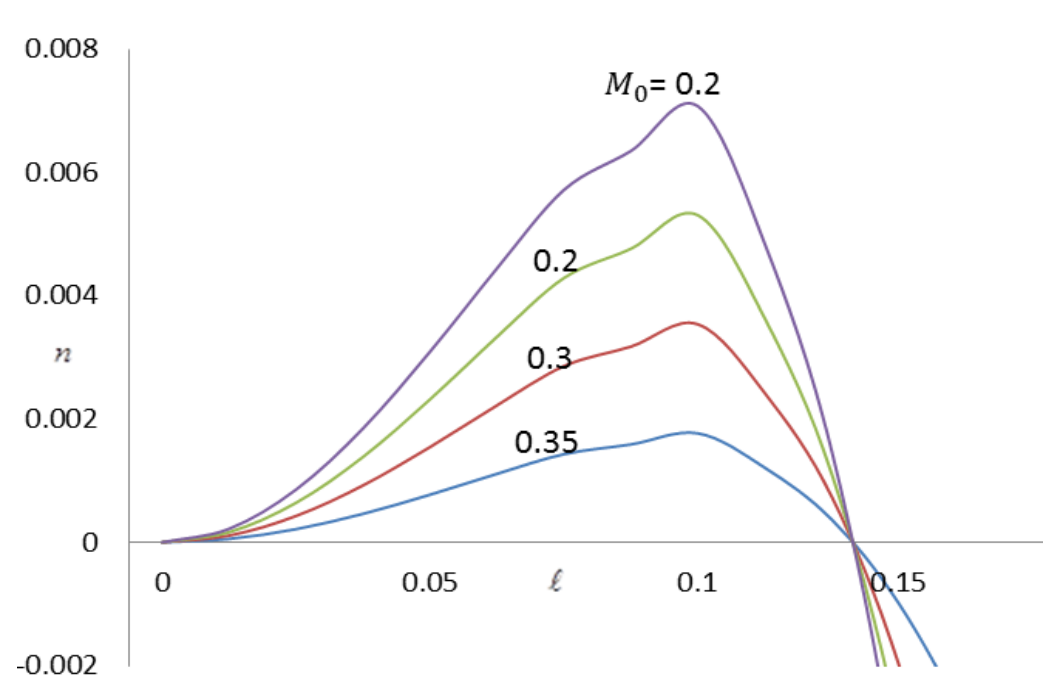

Fig. 3. Variations of Couple-Stress parameter

We have considered $\alpha_{p}=0.1, M_{0}=0.3, \sigma_{p}=4, \beta=3.3 \times 10^{-3}, M=5$ in our sample calculations, and variations in Bond number $\boldsymbol{B}_{1}$. It is observed from Figure 4 that as Bond number, $\boldsymbol{B}_{1}$ is decreased from 0.04 to 0.01 , there is a decrease in critical wavenumber and maximum growth rate. As Bond number and surface tension are inversely related so, an increase in surface tension results into decrease in the growth rate and therefore interface tends to attain stability.

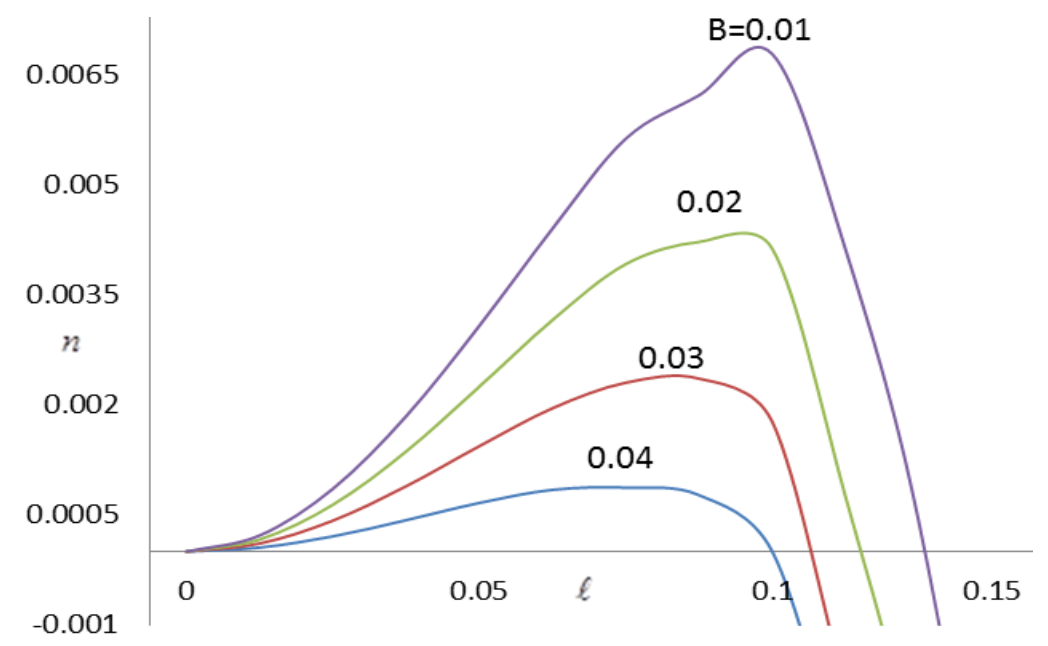

Fig. 4. Variations of Bond number

The effects of porous properties on the instability are investigated by using following input values. The values of the parameters $M_{0}=0.3, \alpha_{p}=0.1, B_{1}=0.02, \beta=3.3 \times 10^{-3}, M=5$ are fixed and vary the value of $\sigma_{p}$. Figure 5 depicts consequences of the calculations, shows that as value of porous parameter $\sigma_{p}$ is increased from 20 to 100 , corresponds to an increase in critical wavelength and decrease in the maximum growth rate, hence exhibits stabilization. This occurs due the resistance offered to the fluid by the solid particles corresponding to the porous layer. 


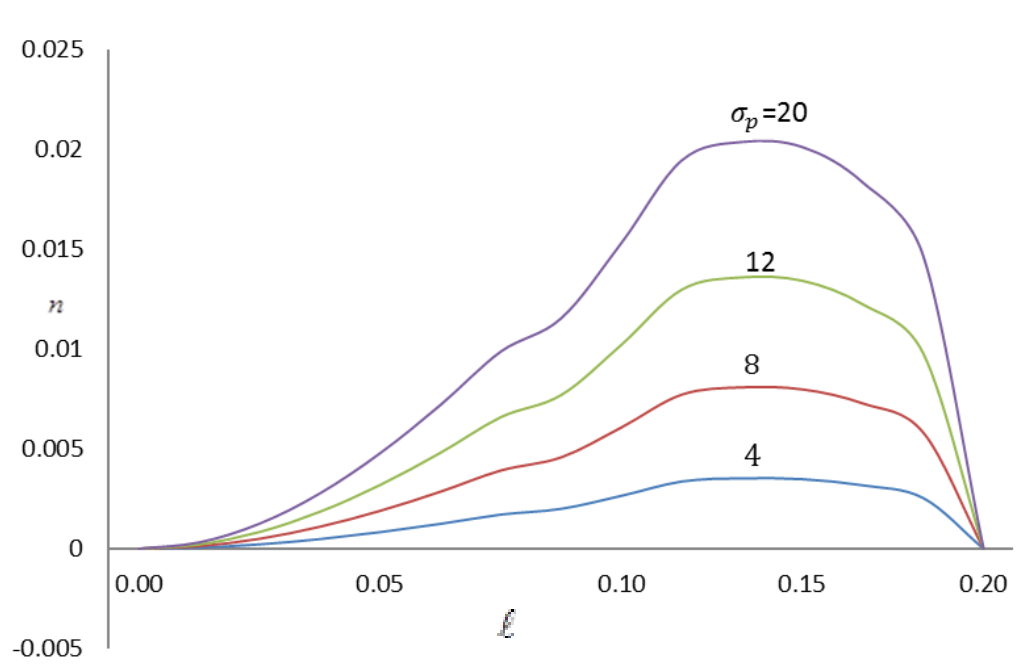

Fig. 5. Variations of Porous Parameter

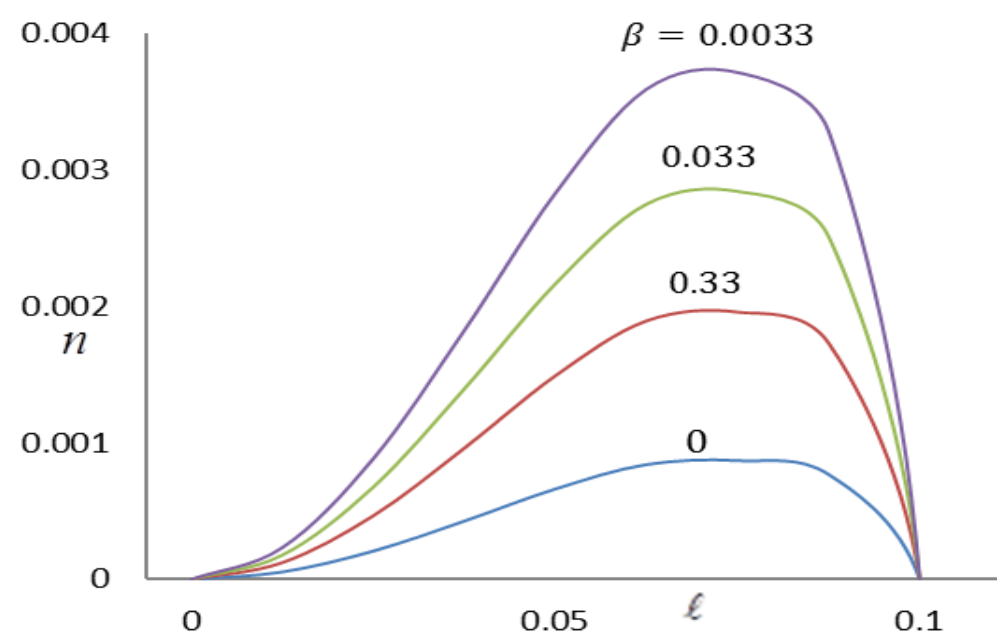

Fig. 6. Variations of Roughness Parameter

In order to analyze boundary roughness effects, other parameter values are fixed as $\alpha_{p}=0.1, \sigma_{p}=4, B_{1}=0.02, M_{0}=0.3, M=5$ and roughness parameter ratio $\beta$ is varied. It is noted that as the value of $\beta$ increases, correspondingly evolution of the interface reduces. This occurs as to overcome the resistance offered by the boundary roughness a part of kinetic energy is transformed into potential energy.

\section{Conclusions}

Linear stability analysis of a two-fluid flow system in a channel is carried out for the fluids which are non-Newtonian possessing various fluid properties. The system under consideration is applied to magnetic field normal to their interface. The equations of motion were derived and then linearized as there occurs an interaction between the couple-stress and hydrodynamic problems at the fluid interface through the stress balance. By the normal mode technique, we calculated the growth rate of the perturbation and analyzed changes in growth rate as a function of nondimensionalized parameters $M, M_{0}, \sigma_{p}, B_{1}, \beta$. From this study, we conclude that an increase in the values of $M, M_{0}, \sigma_{p}, \beta$, the evolution of the interface reduces and the perturbed system tends to attain stability. However, by decreasing the value of the Bond number, $\boldsymbol{B}_{1}$ the growth rate decreases and system becomes more stable. 


\section{Acknowledgement}

The authors (PMG, PIC, GBM) are thankful to Management of KLE Society, University and Principal for their everlasting support and encouragement. The author (KBC) expresses deep sense of gratitude to the UGC and DCE for their kind support in taking up the research.

\section{References}

[1] Helmholtz, H. von. "Uber diskontinierliche Flüssigkeitsbewegungen." Monatsber. Akad. Berlin (1868).

[2] Thomson, William. "XLVI. Hydrokinetic solutions and observations." The London, Edinburgh, and Dublin Philosophical Magazine and Journal of Science 42, no. 281 (1871): $362-377$. https://doi.org/10.1080/14786447108640585

[3] Lamb, Horace. "Hydrodynamics." Victoria University of Manchester, Cambridge University Press, Dover Publications Inc., New York, Printed in the USA, Card Number 46-1891, ISBN: 0486602567 (1945).

[4] Chandrasekhar, Subrahmanyan. "Hydrodynamic and hydromagnetic stability." International Series of Monographs on Physics (1961).

[5] Turner, John Stewart. Buoyancy effects in fluids. Cambridge University Press, 1979.

[6] Babchin, A. J., A. L. Frenkel, B. G. Levich, and G. I. Sivashinsky. "Nonlinear saturation of Rayleigh-Taylor instability in thin films." The Physics of Fluids 26, no. 11 (1983): 3159-3161. https://doi.org/10.1063/1.864083

[7] Malik, S. K., and M. Singh. "Nonlinear focusing and the Kelvin-Helmholtz instability in ferrofluid/nonmagnetic fluid systems." The Physics of Fluids 31, no. 5 (1988): 1069-1073. https://doi.org/10.1063/1.867018

[8] Kundu, Pijush K. Fluid Mechanics. Academic Press, 1990.

[9] De Silva, I. P. D., H. J. S. Fernando, F. Eaton, and D. Hebert. "Evolution of Kelvin-Helmholtz billows in nature and laboratory." Earth and Planetary Science Letters 143, no. 1-4 (1996): 217-231. https://doi.org/10.1016/0012821X(96)00129-X

[10] El-Dib, Yusry O. "Nonlinear stability of Kelvin-Helmholtz waves in magnetic fluids stressed by a time-dependent acceleration and a tangential magnetic field." Journal of Plasma Physics 55, no. 2 (1996): $219-234$. https://doi.org/10.1017/S0022377800018808

[11] Scorer, Richard S. Dynamics of meteorology and climate. John Wiley \& Son Limited, 1997.

[12] Rudraiah, N., R. D. Mathod, and Hameeda Betigeri. "The Rayleigh-Taylor instability of a viscous fluid layer with viscosity stratification." Current Science (1997): 391-395.

[13] Bhatia, P. K., and A. Sharma. "Kelvin-Helmholtz instability of two viscous superposed conducting fluids." Proceedings-National Academy of Sciences India Section B 73, no. 4 (2003): 497-520.

[14] El-Dib, Yusry O., and R. T. Matoog. "Electrorheological Kelvin-Helmholtz instability of a fluid sheet." Journal of Colloid and Interface Science 289, no. 1 (2005): 223-241. https://doi.org/10.1016/i.jcis.2005.03.054

[15] Khan, Aiyub, Neha Sharma, and P. K. Bhatia. "Kelvin-Helmholtz discontinuity in two superposed viscous conducting fluids in a horizontal magnetic field." Thermal Science 12, no. 3 (2008): 103-110. https://doi.org/10.2298/TSCI0803103K

[16] Joshi, Amey S., M. C. Radhakrishna, and N. Rudraiah. "Kelvin-Helmholtz instability in viscoelastic fluids in presence of electro-magnetic fields." Physics of Fluids 23, no. 9 (2011): 094107. https://doi.org/10.1063/1.3637033

[17] Chandrashekara, G., and N. Rudraiah. "Electrorheological Kelvin-Helmholtz Instability at the Interface Between a Nano Structured Porous Layer and Thin Shell with Poorly Conducting Couple Stress Fluid." Fusion Science and Technology 60, no. 1T (2011): 56-63. https://doi.org/10.13182/FST11-A12405

[18] Chavaraddi, Krishna B., N. N. Katagi, V. B. Awati, and Priya M. Gouder. "Effect of boundary roughness on KelvinHelmholtz instability in Couple stress fluid layer bounded above by a porous layer and below by rigid surface." International Journal of Computational Engineering Research 4 (2014): 35-43.

[19] Chavaraddi, Krishna B., Vishwanath B. Awati, and Priya M. "Effect of surface roughness on Kelvin-Helmholtz instability inpresence of magnetic field." International Journal of Engineering Sciences \& Research Technology 4, no. 1 (2015): 525-534.

[20] Sharma, Veena, and Sumit Gupta. "Analytical/numerical investigations of Kelvin-Helmholtz instability of superposed non-viscous fluids in the presence of magnetic field." Research Journal of Engineering and Technology 6, no. 1 (2015): 164-168. https://doi.org/10.5958/2321-581X.2015.00024.0

[21] Chavaraddi, K. B., Awati, V.B., Katagi, N.N and Gouder, P.M. "Effect of Magnetic Field on Kelvin-Helmholtz Instability in a Couple-Stress Fluid Layer Bounded Above by a Porous Layer and Below by a Rigid Surface." Applied Mathematics 7, no. 16 (2016): 2021-2032. https://doi.org/10.4236/am.2016.716164

[22] Shankar, B. M., Shivakumara, I.S and Ng, C.O "Stability of couple stress fluid flow through a horizontal porous layer." Journal of Porous Media 19, no. 5 (2016): 391-404. https://doi.org/10.1615/JPorMedia.v19.i5.20 
[23] Shankar, B. M., Jai Kumar, Shivakumara, I. S. and K. R. Raghunatha. "Stability of natural convection in a vertical non-Newtonian fluid layer with an imposed magnetic field." Meccanica 53, no. 4 (2018): 773-786. https://doi.org/10.1007/s11012-017-0770-6

[24] Liu, Y., Z. H. Chen, H. H. Zhang, and Z. Y. Lin. "Physical effects of magnetic fields on the Kelvin-Helmholtz instability in a free shear layer." Physics of Fluids 30, no. 4 (2018): 044102. https://doi.org/10.1063/1.5004473

[25] Shankar, B. M., Jai Kumar, Shivakumara, I. S. and Naveen Kumar, S. B. "MHD instability of pressure-driven flow of a non-Newtonian fluid." SN Applied Sciences 1, no. 12 (2019): 1523. https://doi.org/10.1007/s42452-019-1557-2

[26] Naveen Kumar, S. B., Shivakumara, I.S. and Shankar, B..M "Exploration of Coriolis force on the linear stability of couple stress fluid flow induced by double diffusive convection." Journal of Heat Transfer 141, no. 12 (2019): 122502. https://doi.org/10.1115/1.4044699

[27] Farahani, Somayeh Davoodabadi, Mahdi Alibeigi, and Hamed Hossienabadi Farahani. "The Uniform Magnetic Field Efficacy on Heat Transfer of Nanofluid Flow in A Flat Tube." Journal of Advanced Research in Numerical Heat Transfer 5, no. 1 (2021): 9-27.

[28] Ch'ng, Kia Chuan, Dmitriy Anatolevich Teryaev, Junior Sarjit Singh Sidhu, and Kok Hwa Yu. "The Impact of Streamwise Steps and Cavities Geometry on Supersonic Turbulent Boundary Layer." CFD Letters 12, no. 11 (2020): 14-26. https://doi.org/10.37934/cfdl.12.11.1426 